\title{
Adaptive Nonlinear Tire-Road Friction Force Estimation for Vehicular Systems Based on a Novel Differentiable Friction Model
}

\author{
Zhi-Jun Fu, Wei-Dong Xie, and Xiao-Bin Ning \\ Vehicle Engineering Research Institute, College of Mechanical Engineering, Zhejiang University of Technology, China
}

Correspondence should be addressed to Zhi-Jun Fu; fuzhijun@zjut.edu.cn

Received 7 February 2015; Revised 8 April 2015; Accepted 28 May 2015

Academic Editor: Raoul van Loon

Copyright (C) 2015 Zhi-Jun Fu et al. This is an open access article distributed under the Creative Commons Attribution License, which permits unrestricted use, distribution, and reproduction in any medium, provided the original work is properly cited.

\begin{abstract}
A novel adaptive nonlinear observer-based parameter estimation scheme using a newly continuously differentiable friction model has been developed to estimate the tire-road friction force. The differentiable friction model is more flexible and suitable for online adaptive identification and control with the advantage of more explicit parameterizable form. Different from conventional estimation methods, the filtered regression estimation parameter is introduced in the novel adaptive laws, which can guarantee that both the observer error and parameter error exponentially converge to zero. Lyapunov theory has been used to prove the stability of the proposed methods. The effectiveness of the estimation algorithm is illustrated via a bus simulation model in the Trucksim software and simulation environment. The relatively accurate tire-road friction force was estimated just by the easily existing sensors signals wheel rotational speed and vehicle speed and the proposed method also displays strong robustness against bounded disturbances.
\end{abstract}

\section{Introduction}

The directional behavior of a vehicle on the road is primarily governed by the forces developed at the tire-road interface and thereby the tire-road friction coefficient. The vehicle control systems especially the active safety control systems such as ABS, traction control, and vehicle stability control have been developed to limit the tire-road interface forces to ensure safe and stable vehicle motion. Although the superior performance of the chassis control systems has been well established under a variety of road conditions and external disturbances, their performance could be greatly enhanced when a real-time estimate of the tire-road friction coefficient is available [1]. The estimation of the tire-road friction force, however, is known to be challenging due to its highly nonlinear dependence on many uncontrollable environmental characteristics such as temperature, tire state, wear, normal force, tire pressure, and road surface conditions, which are difficult to measure directly. The transient nature of the tire-road contact further contributes to the complexities associated with road friction estimations, particularly in the real-time. See [2] for a discussion about the transient nature of tire-pavement interaction and response. In $[3,4]$, a novel wireless piezoelectric tire sensor was used to estimate the friction variations with the tire-road friction coefficient (i.e., dry concrete, wet asphalt, hard snow, and ice). It is well known that the longitudinal tire force at small slip ratio is proportional to the slip ratio. Based on this assumption, a brush model such that the tread elements are modeled as a series of independent springs that undergo longitudinal deformation and resist with a constant longitudinal stiffness is proposed in [5]. A widely used empirical static tire model required for large slip angles and large slip ratios is the so-called Magic Formula proposed by Pacejka and Bakker [6]. The model uses trigonometric functions to describe relationship between slip and friction force generated in the contact between the tire and the road. It is proven that Pacejka model has ability to approximate experimental data. However, as all other static models (such as brush model [5], Burckhardt/Kiencke model [7], and LuGre model [8]) it has significant disadvantage related to inability to describe low slip effects and cannot realize parameter estimate online for 
the nonlinear time-varying parameter. Dugoff's tire model [9] is an alternative to the elastic foundation analytical tire model developed by Fiala [10] for lateral force generation and by Pacejka and Sharp [11] for combined lateral longitudinal force generation. A typical dynamic model that can be used for lateral tire force dynamics is produced by [12]. Unfortunately, these commonly used friction models are discontinuous or piecewise continuous, which bring great difficulty for development of high-performance vehicle safety control system. Moreover, the fixed friction coefficients cannot reflect time-varying friction phenomena accurately in practice.

There are usually two ways to estimate the tire-road friction. One way is that the special sensors that are directly used to measure friction coefficient are utilized as described in [13]. References $[14,15]$ use acoustic sensors that directly attempt to measure friction coefficient. An alternative approach using optical sensors installed at the front bumper of the vehicle to estimate the road surface type is developed in [16]. A method presented in $[17,18]$ uses the drive torque and acceleration signals obtained from a GPS system to estimate the tire forces. However, sensors for friction force measurement are too complex and expensive for practical implementation. For instance, some commercially available optical systems costing over US\$30,000 [19] and the GPS system (e.g., RT3000) from Oxford Technical Solutions are also very expensive. The other way is by the estimator based on the easily measurable signals (such as rotational speed and vehicle speed) to estimate the tire-road friction in an indirect way. Friction force state estimator based on Kalman filter is proposed in [20]. In order to capture variations of the friction model parameters, extended Kalman filter is proposed in [21, 22]. A state estimator based on passivity theory which is able to estimate all state variables and different road conditions is proposed in [23]. In [24] a nonlinear observer for estimating the tire-road force by use of only the known angular wheel velocity is proposed; the simplified motion dynamics of a quarter-vehicle model results in the poor precision. In [25] a nonlinear tire-road friction control based on tire model parameter identification is proposed. The Magic Formula model is proposed as the reference model, and its key parameters are identified online using a constrained hybrid genetic algorithm to describe the evolution of tire-road friction with respect to the wheel slip. All of the aforementioned estimators are based on exact tire-road contact model and therefore are limited by the model accuracy. So, many recent intelligent tire-road friction estimators based on neural network can be found in [26-28]. But they are too complex for practical implantation.

Modeling and compensation for friction effects have been a hot topic in motion control research area in recent years [29-33]. Their basic idea is that if the friction effects in the plant can be accurately modeled, there is an improved potential to design controllers which can avoid the effects of steady-state tracking errors, oscillations, and limit cycles. In order to solve the problem that discontinuous and piecewise continuous friction models are problematic for the development of high-performance continuous controllers, a parameterizable continuously differentiable friction model for friction is proposed in [34]. Motivated by the desire to include dynamic friction models in the control design, numerous researchers have embraced the LuGre friction model. For example, an asymptotic tracking result for square integral disturbances based on the LuGre friction model is proposed in [35]. In [36], Canudas de Wit and Lischinsky proposed observer-based approaches for the LuGre model. Robust adaptive controllers based on LuGre model were also proposed in $[37,38]$. An integrated approach using an intelligent tire-based friction estimator and a brush tire model-based estimator is proposed in [39], which realizes estimation friction for a wider range of excitations. However, the physical brush tire model is not always consistent with the experimental results, because it is based on many assumptions such as the same stiffness of the longitude and lateral, uniform distribution of pressure. Besides, the estimator in [39] is based on the RLS method, which cannot guarantee that the parameter estimations converge to their true values due to the lack considering the parameter error in the updating laws [40].

Summarizing all of the aforementioned friction estimation methods, two issues are still worth mentioning. First, although there are many friction models that can be used to model the friction, but due to the large number of parameters and nonlinear relationship among the existing friction model, which makes them incapable of directly being used for tireroad friction identification, can we design a more suitable model for online adaptive identification friction model? Second, the conventional parameter estimation methods like the gradient method, the RLS methods, and Kalman method are driven by using the tracking error, while the parameter error is not included in the updating laws. It has been proven in [41] that the parameter estimation error is also considered in the updating laws which can improve the error convergence. So, can we design novel updating laws for the friction estimator inspired from [41]? By using the friction compensation in motion control area's research thought, this paper will develop new adaptive nonlinear tire-road friction force estimation vehicular systems based on a new tire-road differentiable friction model. Based on Magic Formula model, a new tire-road friction model with linear parameters is proposed. Then an adaptive observer is developed based on the new tire-road friction model and is used to estimate the friction between tire and road by using the tire rotational speed and vehicle longitudinal speed. A low-pass filtered operation was applied on the longitudinal vehicle dynamics to produce the parameter estimation error without measuring the acceleration. Then a novel online adaptive law using the filtered regression matrix parameter estimation error is proposed. Finally, simulation results based on a realistic bus model constructed in Trucksim verify the proposed estimation algorithm. The main contributions of this paper are as follows:

(i) A new adaptive estimator is proposed to estimate the tire-road friction. The proposed method based on a new tire-road differentiable friction model is more flexible and suitable for online adaptive identification and control with the advantage of more explicit 


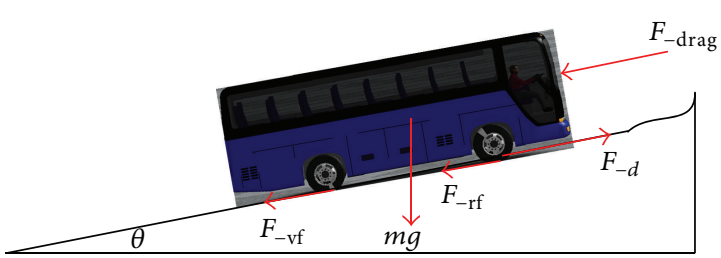

FIgURE 1: Longitudinal dynamics of bus systems.

parameterizable form. Moreover, the developed estimation schemes only require the tire rotational speed and vehicle longitudinal speed.

(ii) Different from the classical estimation methods driven by observer errors, a new estimator driven by the filtered regression matrix estimation parameter error is proposed. It should be pointed out that the estimation parameter instead of observer errors is used to drive the adaptive online updating laws, which can guarantee the observer error convergence to zero and also the estimated parameter convergence to their real value. The convergence of the tracking error is proved via Lyapunov function.

This paper is organized as follows. Section 2 discusses the new tire-road differentiable friction model. Section 3 introduces the adaptive estimator. Section 4 describes the simulation results. Section 5 summarizes the conclusion and finally we have the acknowledgements.

\section{Differentiable Friction Model}

There are usually two types of vehicle friction estimation systems [1]:

(i) systems that utilize longitudinal vehicle dynamics and longitudinal motion measurements,

(ii) systems that utilize lateral vehicle dynamics and lateral motion measurements.

This paper will mainly discuss longitudinal motion based systems. Schematic of the longitudinal dynamics for bus systems is showed in Figure 1.

The longitudinal vehicle dynamics can be presented by

$$
m \dot{v}=F_{-d}-F_{-\mathrm{rf}}-m g \sin \theta-C_{-\mathrm{vf}} v-C v^{2},
$$

where $m$ is the vehicle mass, $v$ is the vehicle speed, $F_{-d}$ is the driving force, $F_{-r f}$ is the rolling friction force, $\theta$ is the road gradient, $F_{-v f}=C_{-v f} v$ is the viscous friction force, and $F_{\text {-drag }}=C v^{2}$ defines the air drag force. Here, we consider only the case of longitudinal dynamics of vehicles to estimate the tire force, where the lateral dynamics are negligible. Thus only the longitudinal force is considered.

The Magic Formula model, with appropriate choice of parameters, can be very effective in representing lateral,

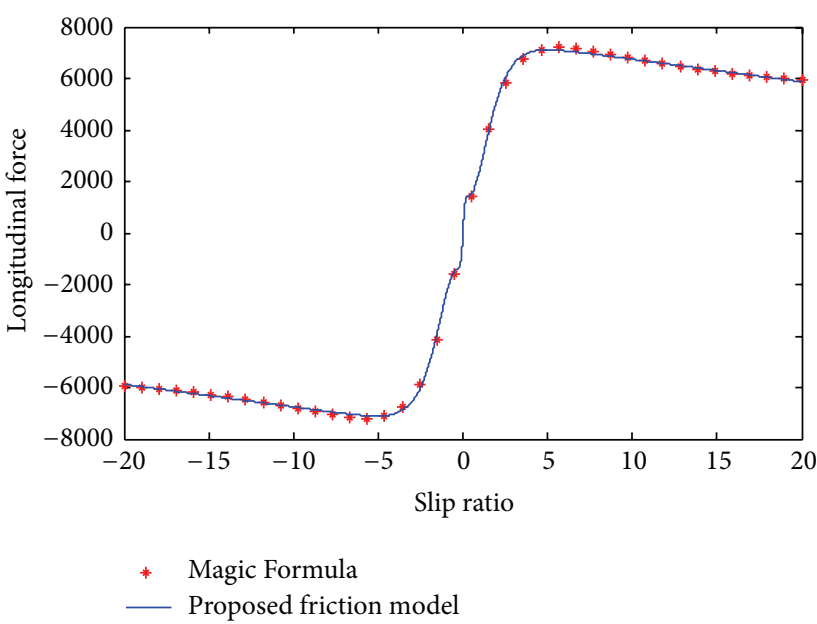

FIGURE 2: Fitting curve of the proposed friction model and the magic model.

longitudinal, and combined tire force generation. So we can get

$$
\begin{aligned}
& F_{-\mathrm{rf}} \\
& \quad=D \sin \left[C \arctan \left\{B s_{x}-E\left(B s_{x}-\arctan \left(B s_{x}\right)\right)\right\}\right] .
\end{aligned}
$$

Unfortunately, due to the large number of parameters and nonlinear relationship among them involved in the Magic Formula, which make it incapable of directly being used for tire-road friction identification, we proposed a new differentiable friction model based on [34]. Assume that $F_{\mathrm{rf}}$ has the following parameterizable form:

$$
\begin{aligned}
F_{-\mathrm{rf}}= & a_{1} \tanh \left(a_{2} s_{x}\right)-a_{7} \tanh \left(a_{3} s_{x}\right)+a_{4} \tanh \left(a_{5} s_{x}\right) \\
& +a_{6} s_{x},
\end{aligned}
$$

where $a_{2}, a_{3}$, and $a_{5}$ mainly determine the profile of the friction effects and $a_{1}, a_{7}, a_{4}$, and $a_{6}$ will be online updated. As can been seen from Figure 2, we can choose proper parameters to make the proposed friction model (3) fit the Magic Formula model by a curve fitting method, so the proposed friction model (3) is effective.

Compared to other friction models, model (3) has a more explicit parameterized form allowing for flexible and suitable adaptive identification and control. Moreover, it has a continuous differential manner while exhibiting static, Stribeck, viscous, and even slip effects.

For the purpose of parameter estimation, we rewrite (1) and (3) in a parameterized form as

$$
\begin{aligned}
m \dot{v} & =-\left(a_{1} \tanh \left(a_{2} s_{x}\right)-a_{7} \tanh \left(a_{3} s_{x}\right)\right. \\
& \left.+a_{4} \tanh \left(a_{5} s_{x}\right)+a_{6} s_{x}\right)+F_{d}-m g \sin \theta-C_{-\mathrm{vf}} v \\
& -C v^{2} .
\end{aligned}
$$

Further (4) can be rewritten as

$$
\dot{v}=\Phi(t) \Theta(t),
$$


where $\Phi(t)=\left[\tanh \left(a_{2} s_{x}\right)-\tanh \left(a_{3} s_{x}\right) \tanh \left(a_{5} s_{x}\right) s_{x}-g\right]$, $\Theta(t)=\left[\begin{array}{lllll}\Theta_{1} & \Theta_{2} & \Theta_{3} & \Theta_{4} & \Theta_{5}\end{array}\right], \Theta_{1}=-(1 / m) a_{1}, \Theta_{2}=$ $(1 / m) a_{7}, \Theta_{3}=-(1 / m) a_{4}, \Theta_{4}=-(1 / m) a_{6}$, and $\Theta_{5}=\sin \theta+$ $(1 / m)\left(C_{-\mathrm{vf}} v+C v^{2}\right)$.

\section{Adaptive Observer}

In order to estimate $\Theta(t)$ with exponential error convergence, we define the filtered variables of $v, \Phi$ as

$$
\begin{aligned}
& k \dot{v}_{f}+v_{f}=v, \quad v_{f}(0)=0, \\
& k \dot{\Phi}_{f}+\Phi_{f}=\Phi, \quad \Phi_{f}(0)=0,
\end{aligned}
$$

where $k$ is the designed filter constant.

Then from (4) and (5) we can get

$$
v_{f}=\frac{v-v_{f}}{k}=\Phi_{f} \Theta .
$$

Further we define the filtered regression matrix $P(t)$ and vector $Q(t)$ as

$$
\begin{aligned}
& \dot{P}(t)=-l P(t)+\Phi_{f}^{T}(t) \Phi_{f}(t), \quad P(0)=0, \\
& \dot{Q}(t)=-l Q(t)+Q_{f}^{T}(t)\left[\frac{\left(v(t)-v_{f}(t)\right)}{k}\right],
\end{aligned}
$$

where $l$ is the designed constant.

From (8) one can get

$$
\begin{aligned}
& P(t)=\int e^{-l(t-r)} \Phi_{f}^{T}(r) \Phi_{f}(r) d r \\
& Q(t)=\int e^{-l(t-r)} \Phi_{f}^{T}(r)\left[\frac{\left(v(r)-v_{f}(r)\right)}{k}\right] d r .
\end{aligned}
$$

Definition 1 (see [42]). A vector or matrix function $\Phi$ is persistently excited (PE) if there exist $\tau>0, \varepsilon>0$ such that $\int_{t}^{t+\tau} \Phi^{T}(r) \Phi(r) d r>\varepsilon I, \forall t \geq 0$.

Remark 2. If the repressor vector $\Phi$ is $\mathrm{PE}$, then $\Phi_{f}$ defined in (6) is PE because $\Phi_{f}$ is the filtered version of $\Phi$ in terms of a minimum strictly proper transfer function $1 /(k s+1)$ in $(6)$ as proved in [42]. Moreover, based on Definition 1 , if $\Phi_{f}$ is $\mathrm{PE}$, the inequality $\int_{t}^{t+\tau} \Phi_{f}^{T}(r) \Phi_{f}(r) d r>\varepsilon I$ is true for all $t>0$, $\varepsilon>0$. Then $\int_{t}^{t+\tau} e^{-l(t-r)} \Phi_{f}^{T}(r) \Phi_{f}(r) d r>\varepsilon I$ holds for all $t>0$, $\varepsilon>0$.

Finally, we denote another auxiliary vector as

$$
W(t)=P(t) \widehat{\Theta}-Q(t),
$$

where $\widehat{\Theta}$ is the estimation of $\Theta(t)$ given by the following adaptive law (13). It is clear that $W(t)$ can be calculated based on (9).

Define the observer error as

$$
e=v-\widehat{v}
$$

Then we can design the observer as

$$
\dot{\hat{v}}=\Phi \widehat{\Theta}+K e .
$$

Now we can design the adaptive law for updating $\widehat{\Theta}$ as

$$
\dot{\widehat{\Theta}}=\Gamma[\Phi \mathrm{e}-\gamma \mathrm{W}],
$$

where $K>0, \gamma>0$, and $\Gamma>0$.

From (4), (11), and (12) we can get

$$
\dot{e}=\dot{v}-\dot{\hat{v}}=\Phi \Theta-\Phi \widehat{\Theta}-K e=-K e+\Phi \widetilde{\Theta}
$$

where $\widetilde{\Theta}=\Theta-\widehat{\Theta}$ is the parameter estimation error.

Theorem 3. Consider system (4) with the observer (12) and parameter adaptive law (13); then the observer error $e=v-\widehat{v}$ and parameter error $\widetilde{\Theta}=\Theta-\widehat{\Theta}$ can exponentially converge to zero.

Proof. Choose a Lyapunov function as

$$
V=\frac{1}{2} e^{2}+\frac{1}{2} \widetilde{\Theta}^{T} \Gamma^{-1} \widetilde{\Theta}
$$

From (11), (12), (13), and (14) and $\dot{\widetilde{\Theta}}=-\dot{\widehat{\Theta}}$ one can get

$$
\begin{aligned}
\dot{V} & =e \dot{e}+\frac{1}{2} \widetilde{\Theta}^{T} \Gamma^{-1} \dot{\widetilde{\Theta}} \\
& =e(-K e+\Phi \widetilde{\Theta})-\widetilde{\Theta}^{T} \Gamma^{-1} \Gamma[\Phi e-\gamma W] \\
& =-K e^{2}+\widetilde{\Theta}^{T} \gamma W .
\end{aligned}
$$

From (7) and (9) one can get

$$
\begin{aligned}
Q(t) & =P(t) \Theta \\
W(t) & =P(t) \widehat{\Theta}-Q(t)=P(t) \widehat{\Theta}-P(t) \Theta \\
& =-P(t) \widetilde{\Theta}
\end{aligned}
$$

Putting (17) into (16) one can get

$$
\dot{V}=-K e^{2}-\gamma \widetilde{\Theta}^{T} P(t) \widetilde{\Theta} .
$$

From Remark 2 we know that $P(t)>0$ for all $t>0$, so $\dot{V}<0$; then the observer error $e=v-\widehat{v}$ and parameter error $\widetilde{\Theta}=\Theta-\widehat{\Theta}$ can exponentially converge to zero.

Remark 4. It should be noted that the online adaptive updating law (13) is different from the conventional estimation methods (e.g., gradient and recursive least square (RLS)). One can find that the second term of (13) is different. As shown in (13), the variable $M$ derived from (10) denotes the estimation parameter not the estimation error. Moreover, we have also given the conclusions that the estimation parameter is used to drive the adaptive updating laws which can guarantee that both the observer error and parameter error exponentially converge to zero. 


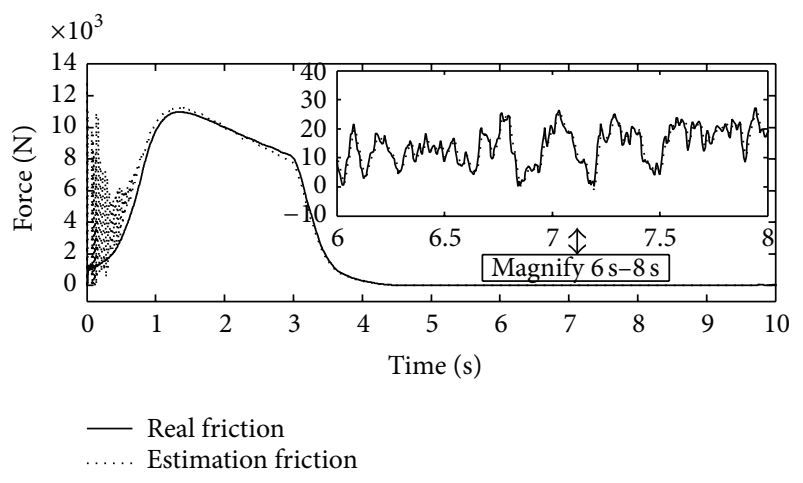

FIGURE 3: Left rear wheel friction force.

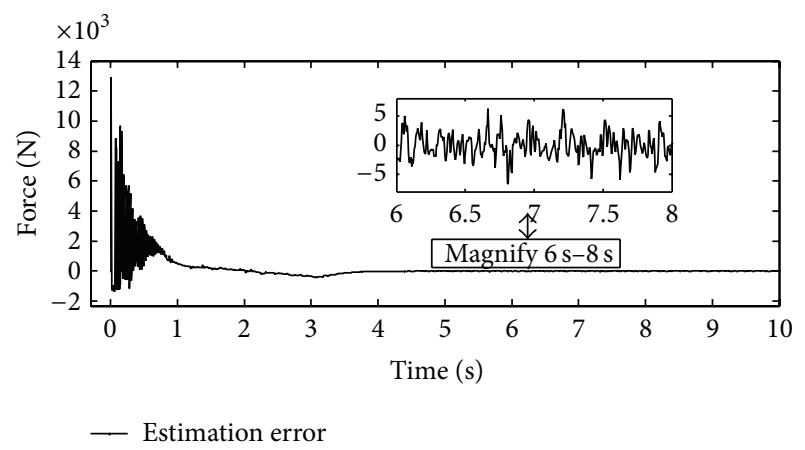

FIgURE 4: Estimation error of the left rear wheel friction force.

\section{Simulation}

A 2WD full bus model was built in the commercial vehicle simulation software Trucksim cooperating with Matlab to verify the estimation performance in the realistic driving maneuvers. The main bus parameters are

$$
\begin{aligned}
& \text { spring mass }=6360 \mathrm{~kg}, \\
& \text { height }=2.92 \mathrm{~m}, \\
& \text { length }=4.49 \mathrm{~m}, \\
& \text { width }=2.35 \mathrm{~m}, \\
& \text { wheel center height } 0.6 \mathrm{~m}, \\
& \text { height for mass }=1.2 \mathrm{~m}, \\
& \begin{array}{l}
\mu=0.85, \\
g=9.8 \mathrm{~m} / \mathrm{s}^{2}, \\
\theta=0 .
\end{array}
\end{aligned}
$$

The bus is driven by the driver controls procedure with the constant target speed $20 \mathrm{~km} / \mathrm{h}$ at a straight long road. The wheel rotational speed and vehicle speed can be obtained from Trucksim. By equation $s=(\omega R-v) / \max (v, \omega R)$ we can get wheel slip; then vehicle speed and wheel slip will be used as the input for the observer. In the simulations, other parameters are given as $k=200, \gamma=0.01, \Gamma=100, a_{2}=200$, $a_{3}=0.01$, and $a_{5}=100$.

Simulation results of the left rear wheel friction force and the estimation error are given in Figures 3 and 4, which

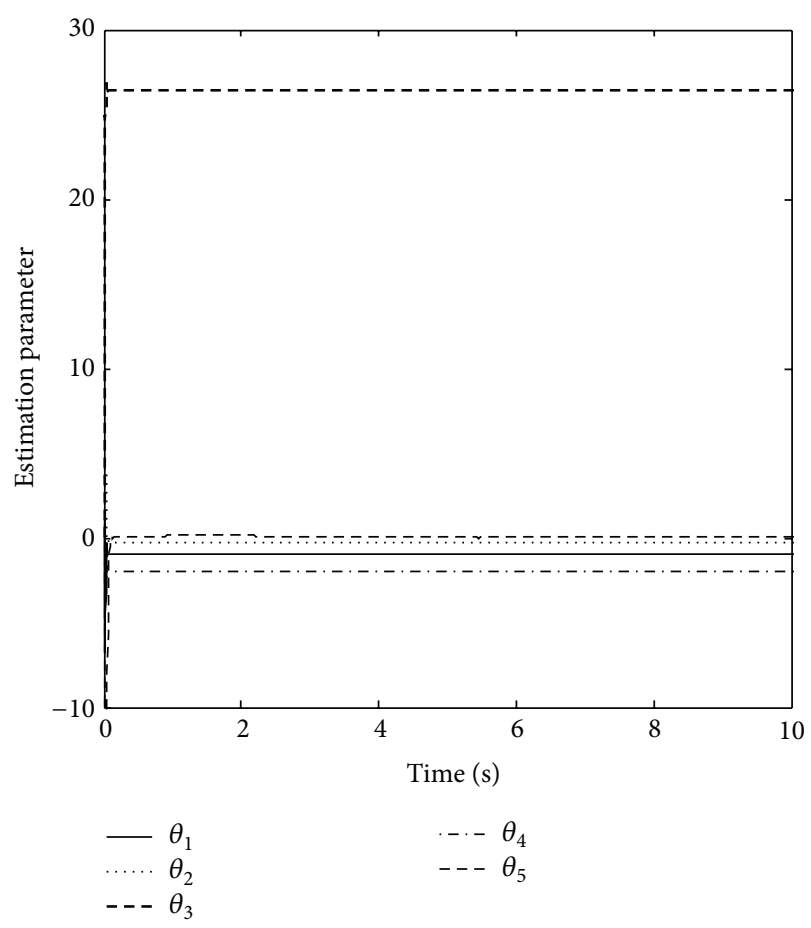

FIGURE 5: Estimated parameters.

illustrate the estimated friction force convergence to the true values with the proposed estimated algorithm except for a little vibration at the vehicle which started before 1 second.

Estimated parameters are shown in Figure 5. From Figure 6, we can see that the observed speed convergence to the target speed is no less than $0.1 \mathrm{~s}$, which further verifies Theorem 3. Figure 7 shows the result of the left rear wheel friction force with the 0.01 white noise power incorporated to the estimator input, and we can see that it also displays better convergence.

\section{Conclusion}

In this paper, we proposed a new friction parameter estimation algorithm for vehicle system. The tire-road friction force can be estimated only by using the longitudinal vehicle velocity and wheel rotational speed. Compared to conventional estimation methods, the obtained parameter estimation error is introduced in the online update laws to guarantee the estimated parameters convergence to the real value. Moreover, the proposed algorithms are proved to be robust to bounded disturbance. Simulation results are presented confirming the validity of the proposed approach. Future work may consider extending the friction estimation method from $2 \mathrm{D}$ case to $3 \mathrm{D}$ case and carrying out the real vehicle experiments to verify the proposed friction parameter estimation methods.

\section{Conflict of Interests}

The authors declare that there is no conflict of interests regarding the publication of this paper. 


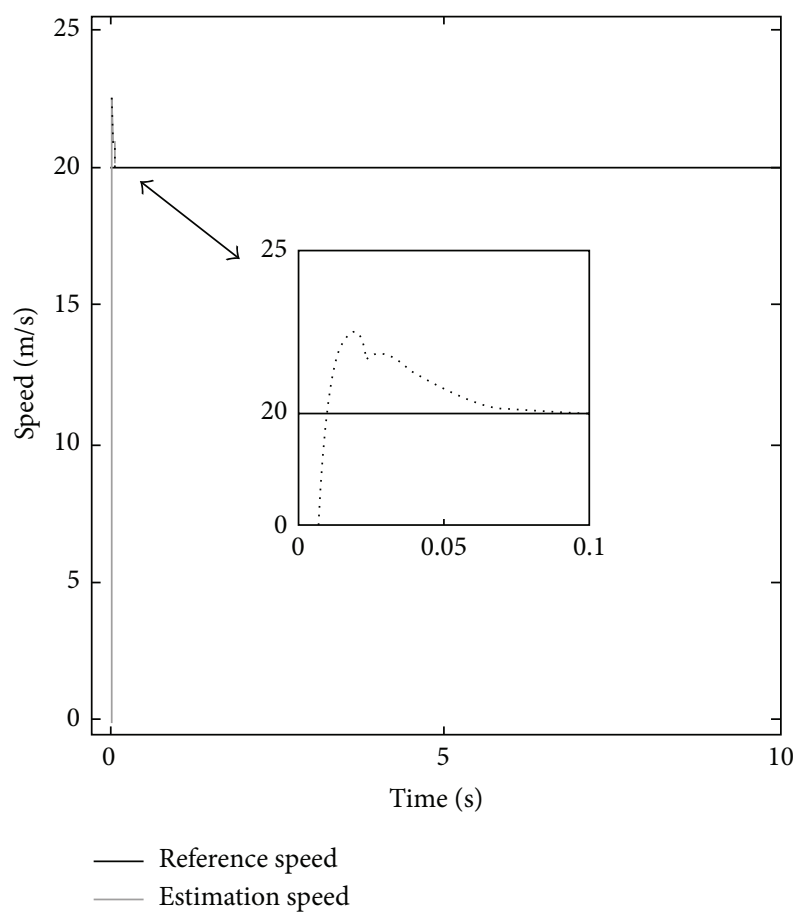

FIGURE 6: Speed convergence.

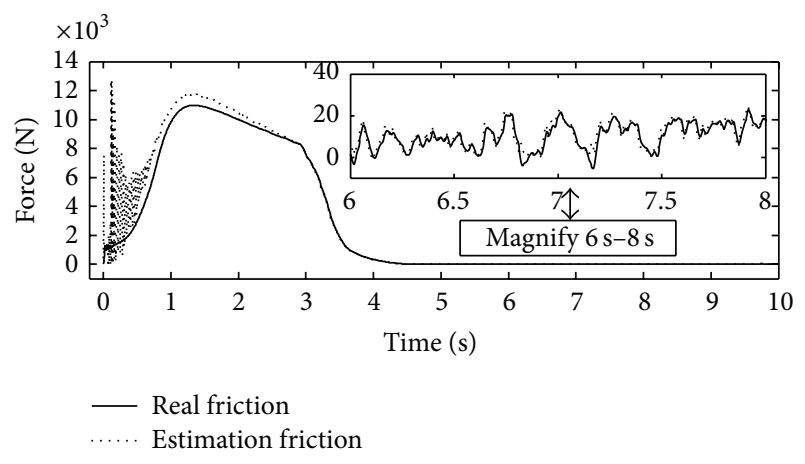

Figure 7: Left rear wheel friction force with bounded disturbance.

\section{Acknowledgments}

This work is supported by the National Natural Science Foundation of China no. 51405436 and no. 51375452, Zhejiang Province Public Technology Research Project no. 2014C31101, and High Intelligence Foreign Expert Project of Zhejiang University of Technology no. 3827102003T.

\section{References}

[1] R. Rajamani, Vehicle Dynamics and Control, Springer, New York, NY, USA, 2012.

[2] R. V. Siddharthan, P. E. Sebaaly, M. El-Desouky, D. Strand, and D. Huft, "Heavy off-road vehicle tire-pavement interactions and response," Journal of Transportation Engineering, vol. 131, no. 3, pp. 239-247, 2005.

[3] G. Erdogan, L. Alexander, and R. Rajamani, "Estimation of tireroad friction coefficient using a novel wireless piezoelectric tire sensor," IEEE Sensors Journal, vol. 11, no. 2, pp. 267-279, 2011.
[4] G. Erdogan, New sensors and estimation systems for the measurement of tire-road friction coefficient and tire slip variables [Ph.D. thesis], University of Minnesota, Minneapolis, Minn, USA, 2009.

[5] J. C. Dixon, Tires, Suspension and Handling, Cambridge University Press, Cambridge, UK, 1991.

[6] H. B. Pacejka and E. Bakker, "The magic formula tyre model," Vehicle System Dynamics, vol. 21, supplement 001, pp. 1-18, 1993.

[7] U. Kiencke and L. Nielsen, Automotive Control Systems: For Engine, Driveline, and Vehicle, Springer, Berlin, Germany, 2000.

[8] N. Patel, C. Edwards, and S. K. Spurgeon, "A sliding mode observer for tyre friction estimation during braking," in Proceedings of the American Control Conference, pp. 5867-5872, Minneapolis, Minn, USA, June 2006.

[9] H. Dugoff, P. S. Fancher, and L. Segal, "Tire performance characteristics affecting vehicle response to steering and braking control inputs," Final Report, Contract CST-460, Office of Vehicle Systems Research, US National Bureau of Standards, 1969.

[10] E. Fiala, "Lateral forces on rolling pneumatic tires," Zeitschrift V.D.I., vol. 96, no. 29, 1954.

[11] H. B. Pacejka and R. S. Sharp, "Shear force development by pneumatic tyres in steady state conditions. A review of modelling aspects," Vehicle System Dynamics, vol. 20, no. 3-4, pp. 121-176, 1991.

[12] P. W. A. Zegelaar, S. Gong, and H. B. Pacejka, "Tyre models for the study of in-plane dynamics," Vehicle System Dynamics, vol. 23, pp. 578-590, 1994.

[13] F. Holzwarth and U. Eichhorn, "Non-contact sensors for road conditions," Sensors and Actuators A: Physical, vol. 37-38, pp. 121-127, 1993.

[14] B. Breuer, U. Eichhorn, and J. Roth, "Measurement of tyre-road friction ahead of the car and inside the tyre," in Proceedings of the International Symposium on Advanced Vehicle Control (AVEC '92), pp. 347-353, 1992.

[15] U. Eichhorn and J. Roth, "Prediction and monitoring of tireroad friction," in Proceedings of the 24th Congress of FISITA, pp. 67-74, London, UK, 1992.

[16] T. Uno, Y. Sakai, J. Takagi, and T. Yamashita, "Road surface recognition method using optical spatial filtering," in Proceedings of the International Symposium on Advanced Vehicle Control (AVEC '94), pp. 509-515, 1994.

[17] J.-O. Hahn, R. Rajamani, and L. Alexander, "GPS-based realtime identification of tire-road friction coefficient," IEEE Transactions on Control Systems Technology, vol. 10, no. 3, pp. 331-343, 2002.

[18] R. Rajamani, G. Phanomchoeng, D. Piyabongkarn, and J. Y. Lew, "Algorithms for real-time estimation of individual wheel tire-road friction coefficients," IEEE/ASME Transactions on Mechatronics, vol. 17, no. 6, pp. 1183-1195, 2012.

[19] Corrsys-Datron optical sensors, http://www.corrsys-datron .com/optical_sensors.htm.

[20] F. Gustafsson, "Slip-based tire-road friction estimation," Automatica, vol. 33, no. 6, pp. 1087-1099, 1997.

[21] J. MatuSko and N. PeriC, "Application of extended Kalmar filter for road condition estimation," in Proceedings of the 10th International Conference on Power Electronics and Motion Control (PEMC '02), Dubrovnik, Croatia, September 2002.

[22] L. R. Ray, "Nonlinear tire force estimation and road friction identification: simulation and experiments," Automatica, vol. 33, no. 10, pp. 1819-1833, 1997. 
[23] C. Canudas-de-Wit and R. Horowitz, "Observers for tire-road contact friction using only wheel angular velocity information," in Proceedings of the 38th IEEE Conference on Decision and Control, vol. 4, pp. 3932-3937, IEEE, Phoenix, Ariz, USA, 1999.

[24] C. Canudas-de-Wit, M. L. Petersen, and A. Shiriaev, "A new nonlinear observer for tire/road distributed contact friction," in Proceedings of the 42nd IEEE Conference on Decision and Control, pp. 2246-2251, Maui, Hawaii, USA, December 2003.

[25] K. Li, J. Cao, and F. Yu, "Nonlinear tire-road friction control based on tire model parameter identification," International Journal of Automotive Technology, vol. 13, no. 7, pp. 1077-1088, 2012.

[26] J. Matuško, I. Petrović, and N. Perić, "Neural network based tire/road friction force estimation," Engineering Applications of Artificial Intelligence, vol. 21, no. 3, pp. 442-456, 2008.

[27] C. Pal, I. Higiwara, S. Morishita, and H. Inoue, "Application of neural networks in real time identification of dynamic structural response and prediction of road friction coefficient $\mu$ from steady state automobile response," in Proceedings of the International Symposium on Advanced Vehicle Control (AVEC '94), pp. 527-532, Tsukuba, Japan, 1994.

[28] J. Matusko, I. Petrovic, and N. Peric, "Application of the RBF neural networks for tire-road friction force estimation," in Proceedings of the IEEE International Symposium on Industrial Electronics (ISIE '03), vol. 2, pp. 701-706, June 2003.

[29] C. Makkar, G. Hu, W. G. Sawyer, and W. E. Dixon, "Lyapunovbased tracking control in the presence of uncertain nonlinear parameterizable friction," IEEE Transactions on Automatic Control, vol. 52, no. 10, pp. 1988-1994, 2007.

[30] D. Zheng, J. Na, X. Ren, G. Herrmann, and S. Longo, "Adaptive control of robotic servo system with friction compensation," in Proceedings of the IEEE 5th International Conference on Robotics, Automation and Mechatronics (RAM '11), pp. 285-290, IEEE, Qingdao, China, September 2011.

[31] J. Na, Q. Chen, X. Ren, and Y. Guo, "Adaptive prescribed performance motion control of servo mechanisms with friction compensation," IEEE Transactions on Industrial Electronics, vol. 61, no. 1, pp. 486-494, 2014.

[32] C. Y. Lai, F. L. Lewis, V. Venkataramanan, X. Ren, S. S. Ge, and T. Liew, "Disturbance and friction compensations in hard disk drives using neural networks," IEEE Transactions on Industrial Electronics, vol. 57, no. 2, pp. 784-792, 2010.

[33] C. Hu, B. Yao, and Q. Wang, "Adaptive robust precision motion control of systems with unknown input dead-zones: a case study with comparative experiments," IEEE Transactions on Industrial Electronics, vol. 58, no. 6, pp. 2454-2464, 2011.

[34] C. Makkar, W. E. Dixon, W. G. Sawyer, and G. Hu, "A new continuously differentiable friction model for control systems design," in Proceedings of the IEEE/ASME International Conference on Advanced Intelligent Mechatronics (AIM '05), pp. 600605, Monterey, Calif, USA, July 2005.

[35] P. Tomei, "Robust adaptive friction compensation for tracking control of robot manipulators," IEEE Transactions on Automatic Control, vol. 45, no. 11, pp. 2164-2169, 2000.

[36] C. Canudas de Wit and P. Lischinsky, "Adaptive friction compensation with partially known dynamic friction model," International Journal of Adaptive Control and Signal Processing, vol. 11, no. 1, pp. 65-80, 1997.

[37] S. Jain, F. Khorrami, N. Ahmad, and S. Sankaranarayanan, "Friction compensation for drives with and without transmission compliance," in Proceedings of the American Control Conference, pp. 2925-2929, Albuquerque, NM, USA, June 1997.
[38] S. Sivakumar and F. Khorrami, "Friction compensation via variable structure control: regulation and low-velocity tracking," in Proceedings of the IEEE International Conference on Control Applications, pp. 645-650, IEEE, Hartford, Conn, USA, October 1997.

[39] K. B. Singh and S. Taheri, "Estimation of tire-road friction coefficient and its application in chassis control systems," Systems Science \& Control Engineering, vol. 3, no. 1, pp. 39-61, 2015.

[40] J. Na, G. Herrmann, X. Ren, M. N. Mahyuddin, and P. Barber, "Robust adaptive finite-time parameter estimation and control of nonlinear systems," in Proceedings of the IEEE International Symposium on Intelligent Control (ISIC '11), pp. 1014-1019, Denver, Colo, USA, September 2011.

[41] V. Adetola and M. Guay, "Finite-time parameter estimation in adaptive control of nonlinear systems," IEEE Transactions on Automatic Control, vol. 53, no. 3, pp. 807-811, 2008.

[42] S. Sastry and M. Bodson, Adaptive Control: Stability, Convergence, and Robustness, Prentice Hall, 1989. 


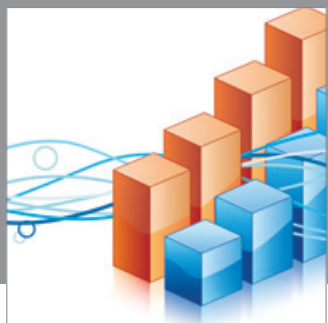

Advances in

Operations Research

mansans

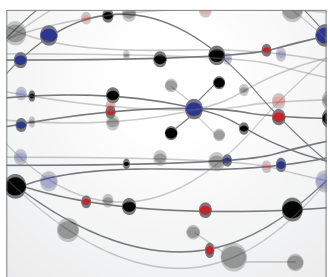

The Scientific World Journal
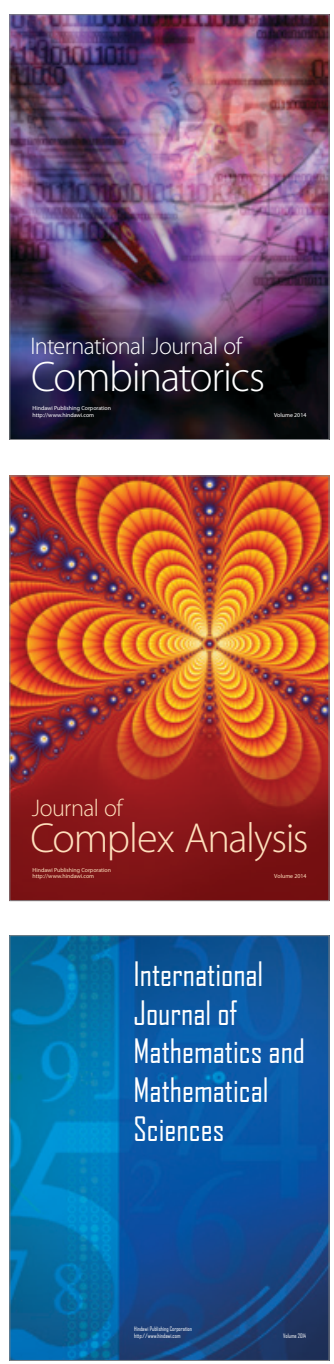
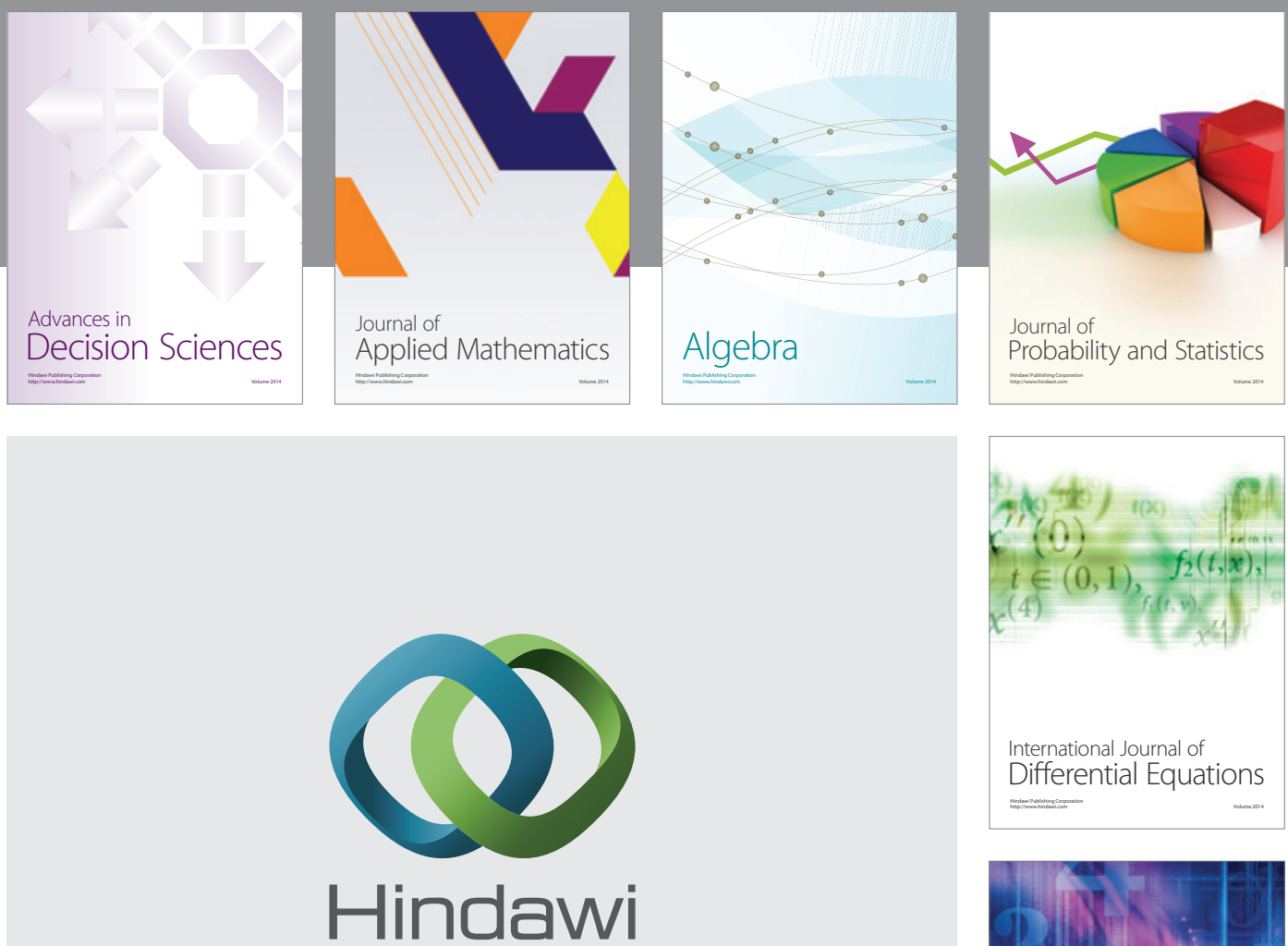

Submit your manuscripts at http://www.hindawi.com
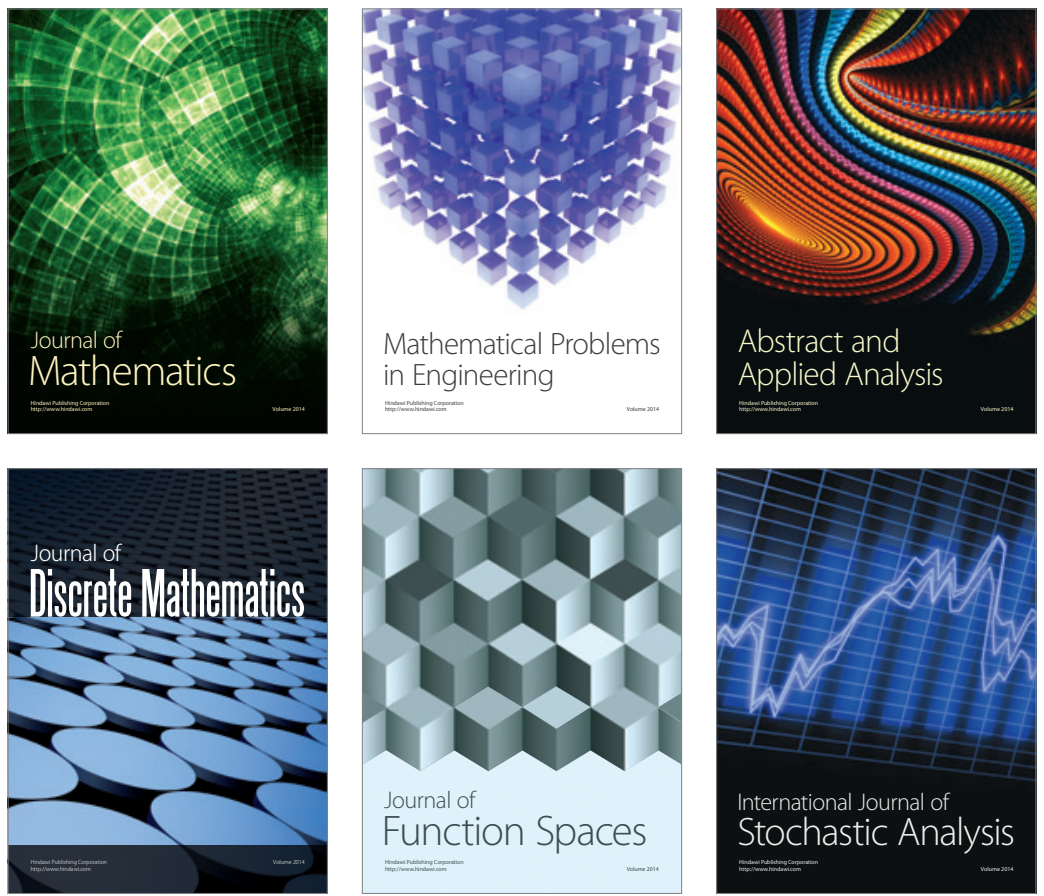

Journal of

Function Spaces

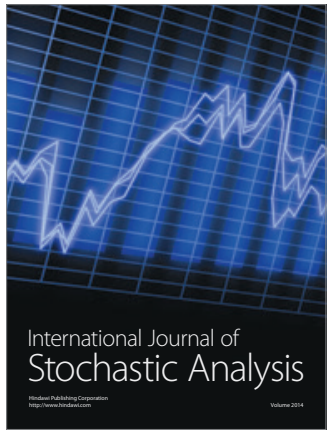

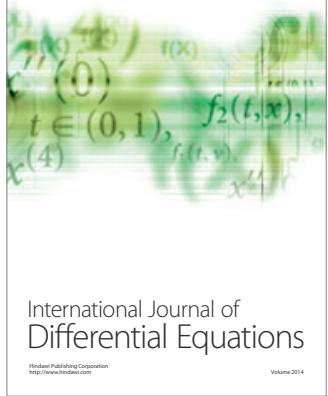
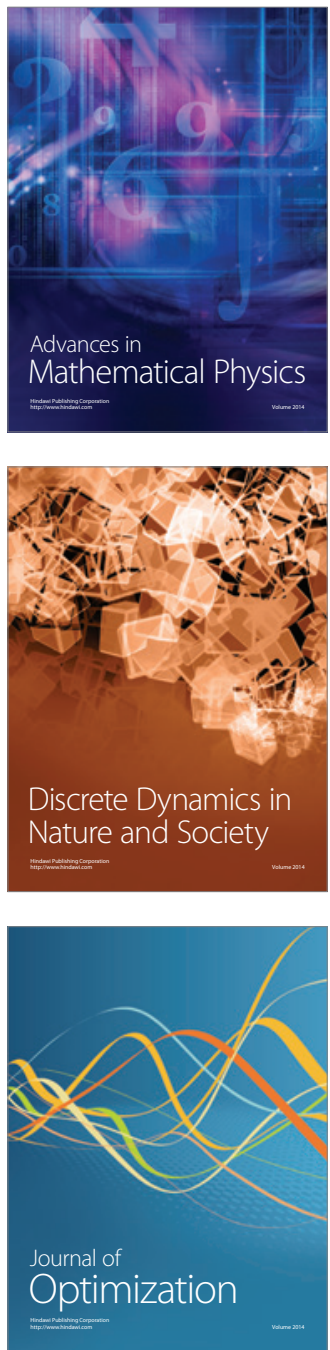